\title{
Output Regulation in Discrete-Time Switched Bimodal Systems Based on Youla Parameterized Stabilizing Controllers
}

\author{
Zhizheng $W_{u^{1}}$ and Foued Ben Amara ${ }^{2}$ \\ ${ }^{1}$ Department of Precision Mechanical Engineering, Shanghai University, 149 Yanchang Road, \\ Shanghai 200072, China \\ ${ }^{2}$ Department of Mechanical and Industrial Engineering, University of Toronto, \\ Toronto, ON, Canada M5S $3 G 8$
}

Correspondence should be addressed to Zhizheng Wu, zhizhengwu@shu.edu.cn

Received 29 May 2012; Revised 28 August 2012; Accepted 3 September 2012

Academic Editor: Jitao Sun

Copyright (c) 2012 Z. Wu and F. Ben Amara. This is an open access article distributed under the Creative Commons Attribution License, which permits unrestricted use, distribution, and reproduction in any medium, provided the original work is properly cited.

\begin{abstract}
Motivated by a class of contact vibration control problems in mechanical systems, this paper considers a regulation problem for discrete-time switched bimodal linear systems where it is desired to achieve output regulation against partially known deterministic and unknown random exogenous signals. First, a set of observer-based Youla parameterized stabilizing controllers is constructed, based on which the regulation conditions for the switched system against the deterministic signals along with an $\mathrm{H}_{2}$ performance constraint against the unknown random signals are derived. Then a corresponding regulator synthesis algorithm is developed based on solving properly formulated linear matrix inequalities. The proposed regulator is successfully evaluated on an experimental setup involving a switched bimodal mechanical system subject to contact vibrations, hence, demonstrating the effectiveness of the proposed regulation approach.
\end{abstract}

\section{Introduction}

Recently, switched control systems have been attracting much attention in the control community since they present problems that are not only academically challenging, but also of practical importance. In the past decades, significant progress has been reported in the literature [1-4] on switched systems and impulsive systems. However, most of the results focus on the stability analysis for such systems. In practice, in addition to stability requirements, there is a need to find controllers that would achieve regulation against reference or disturbance signals. For example, in hard or optical disk drives, maintaining a constant small distance between the read/write head and the disk surface is an important target for 
the design of ultrahigh storage density drives [5-7]. In this case, the read/write head enters into intermittent contact with the disk surface, which results in a switched system regulation problem $[5,6]$. Existing results in the literature on the exact output regulation problem for switched systems have been derived using different approaches and under different assumptions. The case of continuous-time switched systems with preknown switching times and where the model for the exogenous inputs is either completely or partially known is treated in [8]. Regulation in continuous-time piecewise affine (PWA) bimodal systems with completely known reference trajectories and where switching is defined according to a switching surface has been treated in [9] using a generalized error signal. General continuous-time PWA systems have been treated in [10] for the case of perfectly known reference trajectory. A different approach to the regulation problem in continuous-time bimodal linear systems against known exogenous signals was presented by $\mathrm{Wu}$ and Ben Amara in [11-13]. The design of the desired regulator was performed within a set of Youla (or $Q-$-) parameterized regulators for the system. The synthesis algorithms are formulated in sets of bilinear matrix inequalities [11] or linear matrix inequalities [12, 13], respectively.

In this paper, a Youla parameterized controller design approach is proposed for discrete-time switched bimodal linear systems. The goal is to design a controller to achieve regulation against partially known deterministic disturbance or reference signals in the presence of unknown random disturbances. The problem treated in this paper is motivated by the flying height regulation problem in hard or optical disk drives [5-7], where the read/write head is supposed to track, at a small constant distance, the disk surface profile in the presence of known and unknown disturbances and vibrations. The close proximity of the read/write head to the disk surface results in intermittent contact between the two and a bimodal system behaviour. The regulation problem is considered by constructing a set of observer-based parameterized stabilizing controllers that satisfy a sufficient regulation condition for the switched system. A corresponding regulator synthesis algorithm is developed based on solving properly formulated linear matrix inequalities.

The rest of the paper is organized as follows. In Section 2, the general regulation problem for the discrete-time switched bimodal system with deterministic input signals and unknown random signals is presented. In Section 3, a Youla parameterized set of stabilizing switched controllers for the switched system is constructed. In Section 4, regulation conditions for the switched system are presented. In Section 5, a regulator synthesis algorithm is proposed based on solving properly formulated linear matrix inequalities. In Section 6, experimental results are presented to illustrate the effectiveness of the proposed regulators, followed by the conclusion in Section 7 .

\section{The Regulation Problem for Switched Bimodal Systems}

Consider the discrete-time switched bimodal system given by the following state space representation:

$$
\Sigma_{r}:\left\{\begin{array}{l}
x(k+1)=A_{r} x(k)+B_{r} u(k)+D_{r}^{x} d_{r}(k)+F_{r}^{x} d_{w}(k), \quad x(0)=x_{0}, \\
y(k)=C_{r}^{y} x(k)+D_{r}^{y} d_{r}(k)+F_{r}^{y} d_{w}(k), \\
e(k)=C_{r}^{e} x(k)+D_{r}^{e} d_{r}(k)+F_{r}^{e} d_{w}(k), \\
r= \begin{cases}1 & \text { if } e(k)=C_{r}^{e} x(k)+D_{r}^{e} d_{r}(k)+F_{r}^{e} d_{w}(k) \leq \delta, \\
2 \text { if } e(k)=C_{r}^{e} x(k)+D_{r}^{e} d_{r}(k)+F_{r}^{e} d_{w}(k)>\delta,\end{cases}
\end{array}\right.
$$


where $x \in \mathbf{R}^{n}$ is the state vector, $u \in \mathbf{R}$ is the control input, $y \in \mathbf{R}$ is the measurement signal to be fed to the controller, $e \in \mathbf{R}$ is the performance variable to be regulated and is assumed to be measurable, $r \in\{1,2\}$ is the index of the system $\Sigma_{r}$ under consideration at time $k$, and $\delta$ is a constant satisfying $|\delta|>0$. The external signal $d_{w} \in \mathbf{R}^{l}$ represents unknown random signals. The external signal $d_{r} \in \mathbf{R}^{h}$, representing deterministic disturbance and/or reference signals, is also assumed to switch according to the rule given in (2.1). The signal $d_{r}$ is given by:

$$
d_{r}=\left[\begin{array}{lll}
d_{r}^{1} & \cdots & d_{r}^{h}
\end{array}\right]^{T},
$$

where each $d_{r}^{j}$ is of the following form:

$$
d_{r}^{j}(k)=\sum_{i=1}^{k_{j}} c_{i}^{r, j} \cos \left(\omega_{i}^{r, j} k+\phi_{i}^{r, j}\right)+c_{k_{j}+1}^{r, j} \quad r \in\{1,2\}, j=1, \ldots, h,
$$

with known frequencies $\omega_{i}^{r, j}$, and unknown amplitudes $c_{i}^{r, j}$ and phases $\phi_{i}^{r, j}, i=1, \ldots, k_{j}$; $j=1, \ldots, h$. It should be noted that switching is dependent on a time varying switching surface defined by $e(k)=C_{r}^{e} x(k)+D_{r}^{e} d_{r}(k)+F_{r}^{e} d_{w}(k)$, and furthermore the exogenous input $d_{r}$ switches at the same time switching in the plant model takes place. Moreover, $d_{r}$ is discontinuous during switching. The control objective with respect to the switched system (2.1) is to design an output feedback controller to regulate the performance variable $e$ of the switched system against the external input signals $d_{r}$ and $d_{w}$. More specifically, it is desired to achieve exact output regulation against the deterministic exogenous input $d_{r}$ and to minimize the effects of the random input $d_{w}$ to the performance variable $e$, simultaneously.

One of the motivations for the regulation problem presented for the system (2.1) above is the mechanical system shown in Figure 1. In this system, the mass $M$ is supposed to maintain a constant separation with the contact surface $S$ while the latter keeps moving laterally. The system exhibits a bimodal behaviour depending on whether contact between the mass $M$ and the contact surface $S$ takes place. A detailed model for such system is presented in $[5,11]$, which can be found in many practical applications, such as the flying height regulation problem for the read/write head in hard or optical disk drives.

\section{Construction of a Set of Parameterized Output Feedback Controllers}

In this paper, the feedback controllers are obtained by considering Youla $(Q-)$ parameterized output feedback controllers for the switched system (2.1). The construction of such set of controllers proceeds along the same lines as in $[12,13]$. Each controller is expressed as a linear fractional transformation involving a fixed system $J_{r}$ and a proper stable parameter $Q_{r}$ that could be chosen as desired (see Figure 2). The state space representation of the systems $J_{r}$ and $Q_{r}$ are given by the following: 




Figure 1: Example of a mechanical system with switched dynamics.

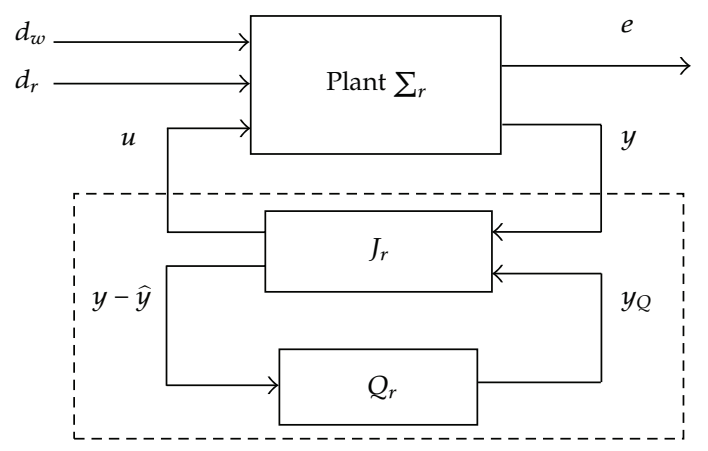

Figure 2: Closed loop system with a $Q$-parameterized controller.

$$
\begin{aligned}
J_{r}:\left\{\begin{array}{l}
\hat{x}(k+1)=\left(A_{r}+L_{r} C_{r}^{y}+B_{r} K_{r}\right) \widehat{x}(k)-L_{r} y(k)+B_{r} y_{Q}(k), \quad \widehat{x}(0)=\widehat{x}_{0}, \\
u(k)=K_{r} \widehat{x}(k)+y_{Q}(k), \\
y(k)-\widehat{y}(k)=y(k)-C_{r}^{y} \widehat{x}(k) .
\end{array}\right. \\
Q_{r}:\left\{\begin{array}{l}
x_{Q}(k+1)=A_{Q} x_{Q}(k)+B_{Q}(y(k)-\widehat{y}(k)), \quad x_{Q}(0)=x_{Q^{\prime}}^{0} \\
y_{Q}(k)=C_{Q_{r}} x_{Q}(k),
\end{array}\right.
\end{aligned}
$$

where $\widehat{x}(k)$ is an estimate of the plant state vector $x(k), \widehat{y}(k)=C_{r}^{y} \widehat{x}(k)$ is an estimate of the plant output $y(k), K_{r}$ and $L_{r}, r \in\{1,2\}$, are state feedback and observer gains, respectively, $x_{Q} \in R^{n_{Q}}, A_{Q}$ is a fixed stability matrix, $B_{Q}$ is a fixed matrix, and matrix $C_{Q_{r}}$ changes with $r \in\{1,2\}$. In this paper, the transfer function of the $Q_{r}$ parameter is considered as the form $Q_{r}(z)=F(z) \sum_{q=1}^{n_{q}} \theta_{r}^{q} z^{1-q}$, where $F(z)$ is a stable function and $\theta_{r}^{q} \in R$. Let $x=\left[x^{T} x_{Q}^{T} \tilde{x}^{T}\right]_{1 \times N^{\prime}}^{T}$ 
$N=2 n+n_{Q}, \tilde{x}(k)=\widehat{x}(k)-x(k)$, then the resulting closed loop system is given by the following state space representation:

$$
\sum_{r}^{c l}:\left\{\begin{array}{l}
x(k+1)=\widehat{A}_{r} X(k)+E_{r}^{d} d_{r}(k)+E_{r}^{w} d_{w}(k), \\
e(k)=C_{r}^{e x} X(k)+D_{r}^{e} d_{r}(k)+F_{r}^{e} d_{w}(k), \\
r=\left\{\begin{array}{l}
1 \text { if } e(k)=C_{r}^{e x} X(k)+D_{r}^{e} d_{r}(k)+F_{r}^{e} d_{w}(k) \leq \delta, \\
2 \text { if } e(k)=C_{r}^{e x} X(k)+D_{r}^{e} d_{r}(k)+F_{r}^{e} d_{w}(k)>\delta,
\end{array}\right.
\end{array}\right.
$$

where

$$
\begin{aligned}
& \widehat{A}_{r}=\left[\begin{array}{ccc}
A_{r}+B_{r} K_{r} & B_{r} C_{Q_{r}} & B_{r} K_{r} \\
0 & A_{Q} & -B_{Q} C_{r}^{y} \\
0 & 0 & A_{r}+L_{r} C_{r}^{y}
\end{array}\right], \quad E_{r}^{d}=\left[\begin{array}{ll}
\left(D_{r}^{x}\right)^{T} & \left(B_{Q} D_{r}^{y}\right)^{T}-\left(D_{r}^{x}+L_{r} D_{r}^{y}\right)^{T}
\end{array}\right]^{T}, \\
& E_{r}^{w}=\left[\begin{array}{ll}
\left(F_{r}^{x}\right)^{T}\left(B_{Q} F_{r}^{y}\right)^{T}-\left(F_{r}^{x}+L_{r} F_{r}^{y}\right)^{T}
\end{array}\right]^{T}, \quad C_{r}^{e x}=\left[\begin{array}{lll}
C_{r}^{e} & 0 & 0
\end{array}\right] .
\end{aligned}
$$

To examine the internal stability of the closed loop system, consider the system (2.1) in the absence of the signals $d_{r}$ and $d_{w}$. The state equation for the resulting system is given by the following:

$$
\left[\begin{array}{c}
x(k+1) \\
x_{Q}(k+1) \\
\tilde{x}(k+1)
\end{array}\right]=\left[\begin{array}{ccc}
A_{r}+B_{r} K_{r} & B_{r} C_{Q_{r}} & B_{r} K_{r} \\
0 & A_{Q} & -B_{Q} C_{r}^{y} \\
0 & 0 & A_{r}+L_{r} C_{r}^{y}
\end{array}\right]\left[\begin{array}{c}
x(k) \\
x_{Q}(k) \\
\tilde{x}(k)
\end{array}\right] .
$$

Based on (3.4), define the following three subsystems:

$$
\begin{gathered}
S 1: x(k+1)=\left[A_{r}+B_{r} K_{r}\right] x(k), \\
S 2: \tilde{x}(k+1)=\left[A_{r}+L_{r} C_{r}^{y}\right] \tilde{x}(k), \\
S 3: x_{Q}(k+1)=A_{Q} x_{Q}(k) .
\end{gathered}
$$

Lemma 3.1. If the switched systems (3.5) and (3.6) are each asymptotically stable under arbitrary switching and $A_{Q}$ is a stability matrix, then the switched system (3.4) is also asymptotically stable under arbitrary switching.

Proof. If the switched system (3.6) is asymptotically stable under arbitrary switching signal, then we have [2]

$$
\|\tilde{x}(k)\|=\|\tilde{\phi}(k, 0) \tilde{x}(0)\| \leq \tilde{c}\|\tilde{x}(0)\| \tilde{a}^{k}
$$


for some $\tilde{c}>0$ and $\tilde{a} \in(0,1)$ where $\tilde{\phi}(k, 0)$ is the state transition matrix of the switched system (3.6). Therefore, $\tilde{x} \rightarrow 0$ as $k \rightarrow \infty$ for any $\tilde{x}(0)$. Similarly, if the system (3.7) is asymptotically stable, then we have

$$
\left\|x_{Q}(k)\right\|=\left\|\phi_{Q}(k, 0) x_{Q}(0)\right\| \leq c_{Q}\left\|x_{Q}(0)\right\| a_{Q^{\prime}}^{k}
$$

for some $c_{Q}>0$ and $a_{Q} \in(0,1)$, where $\phi_{Q}(k, 0)$ is the state transition matrix of the system (3.7). The expression of the state variable $x_{Q}$ in (3.4) is given by:

$$
x_{Q}(k)=\phi_{Q}(k, 0) x_{Q}(0)-\sum_{j=0}^{k-1} \phi_{Q}(k, j) B_{Q} C_{r}^{y} \tilde{x}(j) .
$$

It follows

$$
\begin{aligned}
\left\|x_{Q}(k)\right\| & \leq\left\|\phi_{Q}(k, 0) x_{Q}(0)\right\|+\sum_{j=0}^{k-1}\left\|\phi_{Q}(k, j)\right\|\left\|B_{Q} C_{r}^{y} \tilde{x}(j)\right\| \\
& \leq c_{Q}\left\|x_{Q}(0)\right\| a_{Q}^{k}+c_{Q} \tilde{c}\|\tilde{x}(0)\|\left\|B_{Q} C_{r}^{y}\right\|_{\max } \sum_{j=0}^{k-1} a_{Q}^{k-j} \tilde{a}^{j} .
\end{aligned}
$$

Hence,

$$
\begin{aligned}
& \left\|x_{Q}(k)\right\| \leq c_{Q}\left\|x_{Q}(0)\right\| a_{Q}^{k}+\frac{c_{Q} \tilde{c} a_{Q}\|\tilde{x}(0)\|\left\|B_{Q} C_{r}^{y}\right\|_{\max }}{a_{Q}-\tilde{a}}\left(a_{Q}^{k}-\tilde{a}^{k}\right), \quad \text { if } a_{Q} \neq \tilde{a} \\
& \left\|x_{Q}(k)\right\| \leq c_{Q}\left\|x_{Q}(0)\right\| a_{Q}^{k}+c_{Q} \tilde{c}\|\tilde{x}(0)\|\left\|B_{Q} C_{r}^{y}\right\|_{\max } k a_{Q^{\prime}}^{k}, \quad \text { if } a_{Q}=\tilde{a},
\end{aligned}
$$

which yields

$$
\left\|x_{Q}(k)\right\| \leq\left[c_{Q}\left\|x_{Q}(0)\right\|+\frac{2 c_{Q} \tilde{c}\|\tilde{x}(0)\|\left\|B_{Q} C_{r}^{y}\right\|_{\max }}{\left|a_{Q}-\tilde{a}\right|}\right] a_{m}^{k}, \quad a_{Q} \neq \tilde{a}
$$

where $\left\|B_{Q} C_{r}^{y}\right\|_{\max }$ is the maximum value of $\left\|B_{Q} C_{r}^{y}\right\|$ and $a_{m}=\max \left(a_{Q}, \tilde{a}\right)$. Therefore, we have $x_{Q} \rightarrow 0$ as $k \rightarrow \infty$ for any $x_{Q}(0)$. Similarly, we have that $x \rightarrow 0$ as $k \rightarrow \infty$ for any $x(0)$ in (3.4).

The internal stability of the switched closed loop system (3.4) is then given by the following result. 
Lemma 3.2. The origin is an asymptotically stable equilibrium point for the switched system (3.4) under arbitrary switching if $A_{Q}$ is a stability matrix and there exist matrices $P_{K}=P_{K}^{T}>0, P_{L}=P_{L}^{T}>$ 0 , and matrices $K_{r}, L_{r}, r \in\{1,2\}$, such that

$$
\begin{aligned}
& {\left[A_{r}+B_{r} K_{r}\right]^{T} P_{K}\left[A_{r}+B_{r} K_{r}\right]-P_{K}<0,} \\
& {\left[A_{r}+L_{r} C_{r}^{y}\right]^{T} P_{L}\left[A_{r}+L_{r} C_{r}^{y}\right]-P_{L}<0 .}
\end{aligned}
$$

Proof. Condition (3.14) implies that each of the switched systems $S 1$ and $S 2$ admits a common Lyapunov function. Hence, each of those two systems is asymptotically stable under arbitrary switching. Since $A_{Q}$ is a stability matrix, system $S 3$ is also asymptotically stable. Based on Lemma 3.1, it follows immediately that the switched system (3.4) is asymptotically stable under arbitrary switching.

Remark 3.3. Based on Lemma 3.1 and Lemma 3.2, a Youla parameterized stabilizing controller can be designed by separately considering the subsystems S1, S2, and S3. If feasible parameters $K_{r}$ and $L_{r}$ can be found to make the switched systems $S 1$ and $S 2$ asymptotically stable, then a stable $Q$ parameter of the form $Q(z)=\left(\left(b_{1} z^{s-1}+\cdots+b_{s}\right) /\left(z^{s}+a_{1} z^{s-1}+\cdots+\right.\right.$ $\left.\left.a_{s}\right)\right) \sum_{i=1}^{n_{q}} \theta_{i} z^{1-i}$ could make the overall switched system (3.4) asymptotically stable. The statespace realization of $Q$ can be given as:

$$
A_{Q}=\left[\begin{array}{cccccccc}
0 & \cdots & 0 & 0 & -a_{s} & 0 & 0 & 0 \\
1 & \cdots & 0 & 0 & -a_{s-1} & 0 & 0 & 0 \\
\vdots & \ddots & \vdots & \vdots & \vdots & \vdots & \vdots & \vdots \\
0 & \cdots & 1 & 0 & -a_{2} & 0 & 0 & 0 \\
0 & \cdots & 0 & 1 & -a_{1} & 0 & 0 & 0 \\
0 & \cdots & 0 & 0 & 1 & 0 & 0 & 0 \\
0 & \cdots & 0 & 0 & 0 & \ddots & 0 & 0 \\
0 & \cdots & 0 & 0 & 0 & 0 & 1 & 0
\end{array}\right]_{n_{Q} \times n_{Q}}, \quad B_{Q}=\left[\begin{array}{c}
b_{s} \\
b_{s-1} \\
\vdots \\
b_{2} \\
b_{1} \\
0 \\
\vdots \\
0
\end{array}\right]_{n_{Q} \times 1}
$$

where $\theta=\left[\theta_{1}, \ldots, \theta_{n_{q}}\right]^{T}$ and $n_{Q}=s+n_{q}-1$. It follows that $A_{Q}$ is a fixed stability matrix, $B_{Q}$ is a fixed matrix, and the matrix $C_{Q}$ changes with the parameter vectors $\theta$. A set of stabilizing controllers for switched system (3.4) is hence parameterized by the parameter vector $\theta \in$ $R^{n_{q}}$. The desired controller could be searched among $\theta \in R^{n_{q}}$ according to the regulation conditions to be discussed in Sections 4 and 5.

\section{Regulation Conditions for the Switched Bimodal Systems}

This section presents regulation conditions for the switched closed loop system (3.2) against the external input signals $d_{r}$ and $d_{w}$. Due to the presence of the unknown random signal $d_{w}$, it is not possible to achieve exact output regulation. Consequently, the goal behind the regulator design is to achieve exact output regulation against the deterministic exogenous input $d_{r}$ and minimize the $H_{2}$ norm of the closed loop system relating the input $d_{w}$ to the 
performance variable $e$. It is proposed in this section to achieve these goals by exploiting the flexibility in the selection of the $Q$ parameter in the parameterized set of stabilizing controllers for the switched system. It is well known that the exact regulation conditions for each of the two systems $\Sigma_{1}^{\mathrm{cl}}$ and $\Sigma_{2}^{\mathrm{cl}}$ against $d_{r}$ can be presented in the form of interpolation conditions $[13,14]$ and can be written in the form of linear equations in the unknown parameter vectors $\theta_{r}=\left[\theta_{r}^{1}, \ldots, \theta_{r}^{n_{q}}\right]^{T}, r \in\{1,2\}$, as follows:

$$
A_{\theta_{r}} \theta_{r}+B_{\theta_{r}}=0
$$

where $A_{\theta_{r}}$ and $B_{\theta_{r}}$ are properly formulated matrices. However, using the resulting vector $\theta_{r}$, $r \in\{1,2\}$, the controllers do not guarantee achieving regulation in the switched closed loop system. In the following, additional conditions are placed on the controllers satisfying (4.1) so that switching in the closed loop system would stop after a finite amount of time and exact output regulation would be achieved in the switched closed loop system (3.2) against the deterministic input $d_{r}$. Furthermore, in order to minimize the effects of the unknown random disturbance $d_{w}$ on the performance variable $e$, an $H_{2}$ performance constraint is added in the design of the regulator for the closed loop system. Consider the closed loop system (3.2), and let $T_{w}$ denote the transfer function from $d_{w}$ to $e$ for $\sum_{1}^{\mathrm{cl}}$ when $\delta>0$ or for $\sum_{2}^{\mathrm{cl}}$ when $\delta<0$ and $\left\|T_{w}\right\|_{2}$ denote the $H_{2}$ norm of $T_{w}$, then we have the following regulation condition for the switched system (3.2).

Theorem 4.1. Consider the closed loop system (3.2) subject to bounded inputs $d_{r}$ and $d_{w}$. Let $\kappa=$ $\max _{r \in\{1,2\}, k \geq 0}\left(\left\|d_{r}(k)\right\|^{2}+\left\|d_{w}(k)\right\|^{2}\right) \neq 0$, and $\alpha>0$ a preset constant and $0<\varepsilon \ll 1$. If for $r \in\{1,2\}$, there exist matrices $P>0, X>0, S>0$, and positive scalars $\mu$ and $\beta \leq|\delta|$ such that the following matrix inequalities are satisfied:

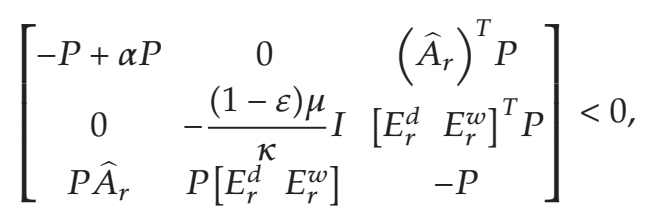

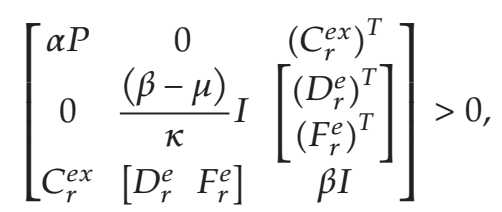

$$
\begin{aligned}
& A_{\theta_{r}} \theta_{r}+B_{\theta_{r}}=0, \quad r= \begin{cases}1 & \text { if } \delta>0, \\
2 & \text { if } \delta<0,\end{cases} \\
& {\left[\begin{array}{ccc}
-X & X \widehat{A}_{r} & 0 \\
\widehat{A}_{r}^{T} X & -X & \left(C_{r}^{e x}\right)^{T} \\
0 & C_{r}^{e x} & -I
\end{array}\right]<0, \quad r= \begin{cases}1 & \text { if } \delta>0, \\
2 & \text { if } \delta<0\end{cases} }
\end{aligned}
$$


Mathematical Problems in Engineering

$$
\begin{gathered}
{\left[\begin{array}{ccc}
X & 0 & X E_{r}^{w} \\
0 & I & F_{r}^{e} \\
\left(E_{r}^{w}\right)^{T} X & \left(F_{r}^{e}\right)^{T} & S
\end{array}\right]>0, \quad r=\left\{\begin{array}{cc}
1 & \text { if } \delta>0 \\
2 & \text { if } \delta<0
\end{array}\right.} \\
\operatorname{Tr}(S)<r^{2}
\end{gathered}
$$

then the state vector $\chi(k)$ and the performance variable $e(k)$ of the switched closed loop system (3.2) are ultimately bounded. Moreover, the switched closed loop system achieves exact output regulation against the deterministic input $d_{r}$ and satisfies $\left\|T_{w}\right\|_{2}<\gamma$ with respect to the unknown random input $d_{w}$.

Proof. Consider the state space representation (3.2) and the quadratic function $V(X)=X^{T} P X$, based on the Schur complement formula on (4.2), for any nonzero vector $\left[\begin{array}{c}x(k) \\ d_{r}(k) \\ d_{w}(k)\end{array}\right] \in R^{2 N+h+1}$, we have

$$
\left[\begin{array}{c}
\chi(k) \\
d_{r}(k) \\
d_{w}(k)
\end{array}\right]^{T}\left[\begin{array}{cc}
\left(\widehat{A}_{r}\right)^{T} P \widehat{A}_{r}-P+\alpha P & \left(\widehat{A}_{r}\right)^{T} P\left[\begin{array}{ll}
E_{r}^{d} & E_{r}^{w}
\end{array}\right] \\
{\left[\begin{array}{ll}
E_{r}^{d} & E_{r}^{w}
\end{array}\right]^{T} P \widehat{A}_{r}} & {\left[\begin{array}{ll}
E_{r}^{d} & E_{r}^{w}
\end{array}\right]^{T} P\left[\begin{array}{ll}
E_{r}^{d} & E_{r}^{w}
\end{array}\right]-\frac{(1-\varepsilon) \mu}{\kappa} I}
\end{array}\right]\left[\begin{array}{c}
x(k) \\
d_{r}(k) \\
d_{w}(k)
\end{array}\right]<0
$$

It follows from the above inequality that for the switched system (3.2):

$$
V(\chi(k+1))-V(\chi(k))<\frac{(1-\varepsilon) \mu}{\kappa}\left[\begin{array}{l}
d_{r}(k) \\
d_{w}(k)
\end{array}\right]^{T}\left[\begin{array}{l}
d_{r}(k) \\
d_{w}(k)
\end{array}\right]-\alpha V(\chi(k))
$$

Hence, $V(\chi(k+1))-V(\chi(k))<0$ holds whenever

$$
\alpha V(\chi(k)) \geq(1-\varepsilon) \mu \geq \frac{(1-\varepsilon) \mu}{\kappa}\left(\left\|d_{r}(k)\right\|^{2}+\left\|d_{w}(k)\right\|^{2}\right)
$$

Consequently, $V(\chi(k))$ cannot ultimately exceed the value $(1-\varepsilon) \mu /(\alpha)$, and we have $\lim _{k \rightarrow \infty} V(\chi(k)) \leq(1-\varepsilon) \mu /(\alpha)$.

Therefore, there exists a finite time $k_{b}$ such that

$$
V(x(k)) \leq \frac{\mu}{\alpha}, \quad \forall k \geq k_{b}
$$

Using the Schur complement formula, inequality (4.3) is equivalent to:

$$
\left[\begin{array}{cc}
\alpha P & 0 \\
0 & \frac{(\beta-\mu)}{\kappa} I
\end{array}\right]-\frac{1}{\beta}\left[\begin{array}{c}
\left(C_{r}^{e x}\right)^{T} \\
\left(D_{r}^{e}\right)^{T} \\
\left(F_{r}^{e}\right)^{T}
\end{array}\right]\left[\begin{array}{lll}
C_{r}^{e x} & D_{r}^{e} & F_{r}^{e}
\end{array}\right]>0
$$


Multiplying from the left by $\left[\begin{array}{c}x(k) \\ d_{r}(k) \\ d_{w}(k)\end{array}\right]^{T}$ and from the right by $\left[\begin{array}{c}x(k) \\ d_{r}(k) \\ d_{w}(k)\end{array}\right]$ yields

$$
\begin{aligned}
e^{2}(k) & <\beta\left(\alpha V(\chi(k))+\frac{(\beta-\mu)}{\kappa}\left[\begin{array}{l}
d_{r}(k) \\
d_{w}(k)
\end{array}\right]^{T}\left[\begin{array}{l}
d_{r}(k) \\
d_{w}(k)
\end{array}\right]\right) \\
& <\beta(\alpha V(\chi(k))+\beta-\mu) .
\end{aligned}
$$

Then, based on (4.11), we have $e^{2}(k)<\beta^{2}, \forall k \geq k_{b}$. Hence, $e(k)$ is ultimately bounded and we have

$$
|e(k)|<\beta, \quad \forall k \geq k_{b}
$$

If $\beta \leq|\delta|$ is satisfied, then after a long enough time $k_{b}$, the output $e(k)$ will always be such that $|e(k)|<\beta \leq|\delta|$ and there will be no more switching. For the case of $\delta>0$, the system $\sum_{1}^{\mathrm{cl}}$ is active for $k \geq k_{b}$. Given that the system $\sum_{1}^{\mathrm{cl}}$ is asymptotically stable and that the parameter vector $\theta_{1}$ satisfies the regulation condition in (4.1), then exact output regulation for the switched system is achieved against the deterministic input $d_{r}$. Furthermore, based on (4.5)-(4.7), it is easy to obtain $\operatorname{Tr}\left(\left(F_{r}^{e}\right)^{T} F_{r}^{e}+\left(E_{r}^{w}\right)^{T} X E_{r}^{w}\right)<\gamma^{2}$, where $\widehat{A}_{r}^{T} X \widehat{A}_{r}-X+\left(C_{r}^{e x}\right)^{T} C_{r}^{e x}<0$ and $X>0$, which implies $\left\|T_{w}\right\|_{2}<\gamma$ [15]. The analysis is similar for the case of $\delta<0$.

Remark 4.2. The main reason for considering common Lyapunov functions as opposed to multiple Lyapunov functions in (3.14), (4.2) and (4.3) is as follows. The existence of a common Lyapunov function for each of the switched systems $S 1$ and $S 2$ in (3.14) implies the existence of a common Lyapunov function in (4.2) and (4.3) for the system (3.4). The less conservative regulation conditions (4.2) and (4.3) can be formulated using multiple Lyapunov functions as in the approach presented in [13]. However, as outlined in Section 5, the use of a common Lyapunov function allows for the regulation conditions to be transformed into linear matrix inequalities (LMIs) in the regulator synthesis algorithm using an appropriate congruence transformation so that a global optimal solution can be found for the controller. The use of multiple Lyapunov functions would have made such transformation very difficult. Moreover, the synthesis algorithm normally would in that case be formulated using bilinear matrix inequalities, which would typically yield a conservative local solution for the controller.

Remark 4.3. In Theorem 4.1, the $H_{2}$ performance constraint applies only to the closed loop system that is active when regulation against the deterministic exogenous input is achieved, that is, the closed loop system operating in mode 1 if $\delta>0$ and in mode 2 if $\delta<0$. This is due to the fact that if conditions (4.2) and (4.3) are satisfied, then switching in the closed loop system stops after a finite amount of time. Consequently, it is enough to apply the additional $\mathrm{H}_{2}$ performance constraint only to the closed loop system that is active when switching stops after a finite amount of time. Optimal performance for switched systems defined based on other performance measures, as presented for example in [16, 17], can also be considered. However, that will introduce conservativeness in the final solution since continuous switching is assumed to take place in those approaches, which is not the case for the systems considered in this paper. 


\section{Regulator Synthesis}

The synthesis method aims at finding the proper $K_{r}, L_{r}$, and $C_{Q_{r}}$ such that conditions (4.2)(4.7) are satisfied. The main idea behind the regulator synthesis is to first design the gains $K_{r}$ and $L_{r}$ based on (3.14). Since $A_{Q}$ and $B_{Q}$ in (4.2) and (4.5) are fixed, then it is only necessary to find the matrices $C_{Q_{r}}$ such that the regulation conditions (4.2)-(4.7) are satisfied. Partition



$$
\begin{aligned}
& M_{r}\left(\Omega_{1}, \Omega_{2}, \Omega_{3}, C_{Q_{r}}\right) \\
& \quad=\left[\begin{array}{c|c}
{\left[A_{r}+B_{r} K_{r}\right] \Omega_{1}} & \Omega_{2}\left[\begin{array}{cc}
A_{Q} & -B_{Q} C_{r} \\
0 & A_{r}+L_{r} C_{r}
\end{array}\right]-\left[A_{r}+B_{r} K_{r}\right] \Omega_{2}+\left[B_{r} C_{Q_{r}} B_{r} K_{r}\right] \\
\hline 0 & \Omega_{3}\left[\begin{array}{cc}
A_{Q} & -B_{Q} C_{r} \\
0 & A_{r}+L_{r} C_{r}
\end{array}\right]
\end{array}\right] .
\end{aligned}
$$

Multiplying (4.2) from the left side by $\operatorname{diag}(\Omega, I, \Omega)$ and from the right side by $\operatorname{diag}(\Omega, I, \Omega)^{T}$ yields

$$
\left[\begin{array}{ccc}
(\alpha-1)\left[\begin{array}{cc}
\Omega_{1} & 0 \\
0 & \Omega_{3}
\end{array}\right] & 0 & M_{r}^{T}\left(\Omega_{1}, \Omega_{2}, \Omega_{3}, C_{Q_{r}}\right) \\
0 & -\frac{(1-\varepsilon) \mu}{\kappa} I & {\left[\begin{array}{cc}
E_{r}^{d} & E_{r}^{w}
\end{array}\right]^{T}\left[\begin{array}{cc}
I & 0 \\
\Omega_{2}^{T} & \Omega_{3}
\end{array}\right]} \\
M_{r}\left(\Omega_{1}, \Omega_{2}, \Omega_{3}, C_{Q_{r}}\right) & {\left[\begin{array}{cc}
I & \Omega_{2} \\
0 & \Omega_{3}
\end{array}\right]\left[\begin{array}{ll}
E_{r}^{d} & E_{r}^{w}
\end{array}\right]} & -\left[\begin{array}{cc}
\Omega_{1} & 0 \\
0 & \Omega_{3}
\end{array}\right]
\end{array}\right]<0 .
$$

Multiplying (4.3) from the left side by $\operatorname{diag}(\Omega, I, I)$ and from the right side by $\operatorname{diag}\left(\Omega^{T}, I, I\right)$ yields

$$
\left[\begin{array}{ccc}
\alpha\left[\begin{array}{cc}
\Omega_{1} & 0 \\
0 & \Omega_{3}
\end{array}\right] & 0 & {\left[\begin{array}{cc}
\Omega_{1} & 0 \\
-\Omega_{2}^{T} & I
\end{array}\right]\left(C_{r}^{e x}\right)^{T}} \\
0 & \frac{(\beta-\mu)}{\kappa} I & {\left[\begin{array}{c}
\left(D_{r}^{e}\right)^{T} \\
\left(F_{r}^{e}\right)^{T}
\end{array}\right]} \\
C_{r}^{e x}\left[\begin{array}{cc}
\Omega_{1} & -\Omega_{2} \\
0 & I
\end{array}\right] & {\left[\begin{array}{ll}
D_{r}^{e} & F_{r}^{e}
\end{array}\right]} & \beta I
\end{array}\right]>0 .
$$


Similarly, we partition $X$ in (4.5) as $\left(\begin{array}{cc}{\left[X_{1}\right]_{n \times n}} & X_{2} \\ X_{2}^{T} & X_{3}\end{array}\right)$ and define $\Psi_{1}=X_{1}^{-1}, \Psi_{2}=X_{1}^{-1} X_{2}, \Psi_{3}=$ $X_{3}-X_{2}^{T} X_{1}^{-1} X_{2}$ and $\Psi=\left[\begin{array}{cc}\Psi_{1} & 0 \\ -\Psi_{2}^{T} & I\end{array}\right]$. Multiplying (4.5) from the left side by $\operatorname{diag}(\Psi, \Psi, I)$ and from the right side by $\operatorname{diag}\left(\Psi^{T}, \Psi^{T}, I\right)$ yields the following:

$$
\left[\begin{array}{ccc}
-\left[\begin{array}{cc}
\Psi_{1} & 0 \\
\mathbf{0} & \Psi_{3}
\end{array}\right] & M_{r}\left(\Psi_{1}, \Psi_{2}, \Psi_{3}, C_{Q_{r}}\right) & 0 \\
M_{r}^{T}\left(\Psi_{1}, \Psi_{2}, \Psi_{3}, C_{Q_{r}}\right) & -\left[\begin{array}{cc}
\Psi_{1} & 0 \\
0 & \Psi_{3}
\end{array}\right] & {\left[\begin{array}{cc}
\Psi_{1} & 0 \\
-\Psi_{2}^{T} & I
\end{array}\right]\left(C_{r}^{e x}\right)^{T}} \\
0 & C_{r}^{e x}\left[\begin{array}{cc}
\Psi_{1} & -\Psi_{2} \\
0 & I
\end{array}\right] & -I
\end{array}\right]<0, \quad r=\left\{\begin{array}{l}
1 \text { if } \delta>0, \\
2 \text { if } \delta<0 .
\end{array}\right.
$$

Multiplying (4.6) from the left side by $\operatorname{diag}(\Psi, I, I)$ and from the right side by $\operatorname{diag}\left(\Psi^{T}, I, I\right)$ yields

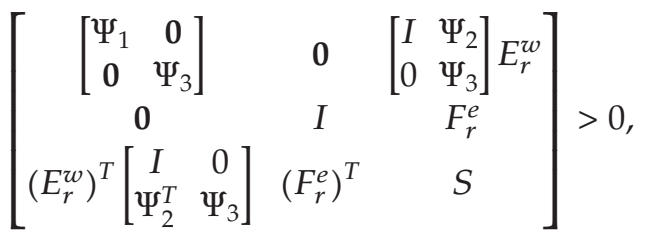

In the following, a regulator synthesis algorithm is presented to obtain $C_{Q_{r}}$ matrices that simultaneously minimize the $\mathrm{H}_{2}$ performance $\gamma$ and satisfy the regulation conditions in Theorem 4.1. The main steps in the proposed regulator synthesis procedure are summarized below.

(1) Design of an internally stabilizing controller: design the controller parameters $K_{r}$ and $L_{r}$ to make the switched closed loop system (3.2) internally stable by solving (3.14) for the unknown matrices $P_{K}=P_{K}^{T}>0, P_{L}=P_{L}^{T}>0$ and matrices $K_{r}, L_{r}$, $r \in\{1,2\}$.

(2) Find an upper bound $\alpha_{m}$ on the value of $\alpha$ in (5.2) with unknown $\Omega_{1}>0, \Omega_{2}$, $\Omega_{3}>0$, and $C_{Q_{r}}$, which is a generalized eigenvalue problem. Select a value of $\alpha$ in the interval $\alpha \in\left(0, \alpha_{m}\right)$.

(3) Substitute $|\delta|$ for $\beta$ in (5.3).

(4) Minimize $\operatorname{Tr}(S)$ subject to (4.4), (5.2), (5.3), (5.4) and (5.5), in the unknown $\mu, \Omega_{1}$, $\Omega_{2}, \Omega_{3}, \Psi_{1}, \Psi_{2}, \Psi_{3}, S$, and $C_{Q_{r}}$ with known $\alpha$.

Note that (5.2) and (5.3) are linear in the unknown parameters $\beta, \mu, \Omega_{1}, \Omega_{2}, \Omega_{3}$ and $C_{Q_{r}}$ only if $\alpha$ is prefixed. The value of $\alpha$ can be selected using a linear search in the interval $\alpha \in$ $\left(0, \alpha_{m}\right)$ in order to solve the optimization problem in Step 4 . In the following, an experimental example is presented to show the effectiveness of the proposed regulator synthesis method.

\section{Experimental Evaluation}

The experimental setup represents a mechanical system subject to contact vibrations and is motivated by the flying height regulation problem in hard or optical disk drives [5-7]. 


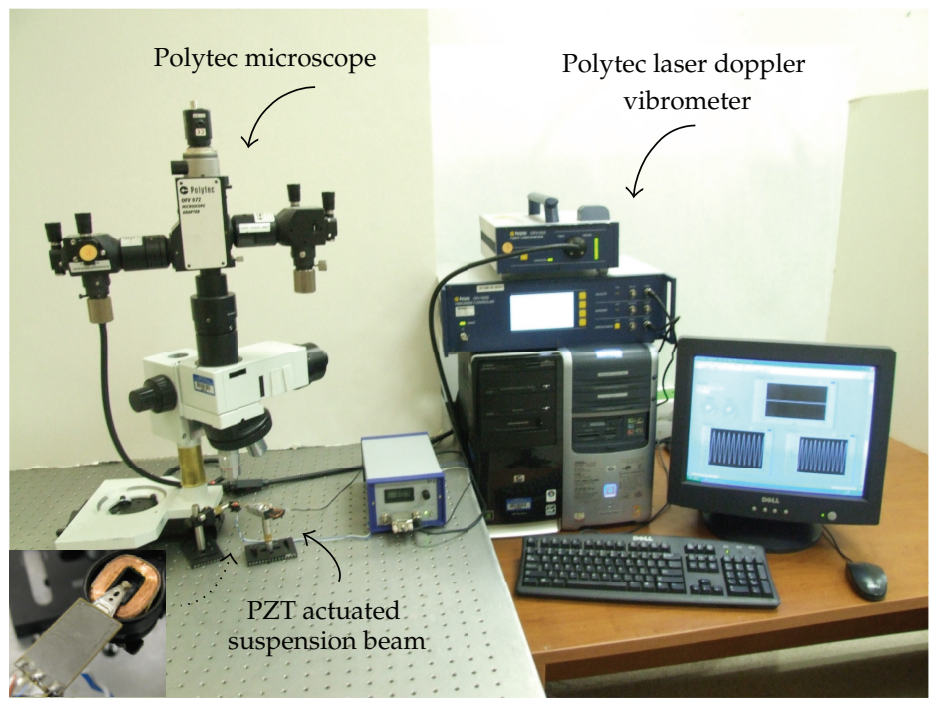

Figure 3: Images of the experimental setup.

The experimental setup consists mainly of a flexible beam subject to intermittent contact with a fictitious contact surface. The system has two modes of operation, one corresponding to the case of noncontact between the beam tip and the contact surface, and the second corresponds to the case when contact takes place. The control goal in the experiment is to maintain a constant separation between the tip of the flexible beam and the contact surface in the presence of external disturbances and switching between the noncontact mode and the contact mode of the system. Images of the experimental setup are shown in Figure 3. A flexible beam (the suspension beam in a hard disk drive) is actuated using a multilayer piezoelectric actuator (PZT). A Laser Doppler Vibrometer (LDV) provides realtime measurements of the position and velocity of the beam tip. A small-size permanent magnet is attached to the tip of the beam. In the experimental setup, contact between the beam tip and a contact surface is simulated by generating a vertical contact force using an electromagnetic actuator underneath and applying the generated force to the beam tip. The forces representing deterministic disturbances and random disturbances are also produced using the electromagnetic actuator. The sampling period in the experimental setup is of $1 \mathrm{KHz}$. The two subsystem models corresponding to the contact mode and the noncontact mode are obtained using system identification method. The regulator is designed so that the tip of the suspension beam tracks a contact surface $s_{c}=(5 \sin (240 \pi t)-60) \times 10^{-6} \mathrm{~m}$ at a desired separation height of $60 \times 10^{-6} \mathrm{~m}$. The output $y$ and the performance variable $e$ are identical and are defined to be the difference between the actual separation and the desired separation height. The state space representation as in (2.1) is given as

$$
\begin{array}{cc}
A_{1}=\left[\begin{array}{ll}
0 & -0.8735 \\
1 & -0.5645
\end{array}\right], & A_{2}=\left[\begin{array}{ll}
0 & -0.9313 \\
1 & -0.2459
\end{array}\right], \\
B_{1}=\left[\begin{array}{l}
1.8045 \\
0.9125
\end{array}\right], & B_{2}=\left[\begin{array}{l}
2.1601 \\
1.0036
\end{array}\right],
\end{array}
$$




$$
\begin{gathered}
D_{1}^{x}=\left[\begin{array}{ll}
0.2021 & 0 \\
0.2010 & 0
\end{array}\right], \quad F_{1}^{x}=\left[\begin{array}{l}
0.2021 \\
0.2010
\end{array}\right], \\
D_{2}^{x}=\left[\begin{array}{ll}
0.2267 & 0 \\
0.2187 & 0
\end{array}\right], \quad F_{2}^{x}=\left[\begin{array}{l}
0.2267 \\
0.2187
\end{array}\right], \\
C_{1}^{e}=C_{1}^{y}=\left[\begin{array}{ll}
0 & 1
\end{array}\right], \quad C_{2}^{e}=C_{2}^{y}=\left[\begin{array}{ll}
0 & 1
\end{array}\right], \\
d_{1}=\left[\begin{array}{c}
0.5 \cos \left(240 \pi k+\frac{3 \pi}{4}\right)+0.4 \sin (240 \pi k)+0.3 \cos (240 \pi k) \\
0.5 \sin (240 \pi k)
\end{array}\right] \text { volts, } \\
d_{2}=\left[\begin{array}{c}
0.5 \cos \left(240 \pi k+\frac{3 \pi}{4}\right) \\
0.5 \sin (240 \pi k)
\end{array}\right] \text { volts. }
\end{gathered}
$$

The switching rule is defined as $r=\left\{\begin{array}{l}1 \text { if } e \leq-6 \text { volts } \\ 2 \text { if } e>-6 \text { volts }\end{array}\right.$. To construct a parameterized set of stabilizing controllers, the gains $K_{r}, L_{r}, r \in\{1,2\}$, designed based on (3.14) are given by:

$$
\begin{aligned}
& K_{1}=[-159.1746504 .2805] \times 10^{-3}, \quad K_{2}=[-126.6187407 .4727] \times 10^{-3}, \\
& L_{1}=[873.4887564 .5477]^{T} \times 10^{-3}, \quad L_{2}=[931.2618245 .8963]^{T} \times 10^{-3} .
\end{aligned}
$$

The set of controllers to be designed is obtained by considering $Q_{r}$ parameters of the form $Q_{r}=\left((0.46 z+0.45) /\left(z^{2}-1.4 z+0.9\right)\right)\left(\left(\theta_{r}^{1} / z\right)+\left(\theta_{r}^{2} / z^{2}\right)+\left(\theta_{r}^{3} / z^{3}\right)+\left(\theta_{r}^{4} / z^{4}\right)\right) \times 10^{3}$. Since $\delta<0$, condition (4.1) needs to consider only the system $\Sigma_{2}$ and yields:

$$
A_{\theta_{2}}=\left[\begin{array}{cccc}
0.8619 & 8.8250 & 12.0044 & 8.6766 \\
11.9739 & 8.1385 & -0.1084 & -8.2966
\end{array}\right] \times 10^{3}, \quad B_{\theta_{2}}=\left[\begin{array}{c}
305.1971 \\
-261.1491
\end{array}\right] \times 10^{-3}
$$

First, without considering the $\mathrm{H}_{2}$ performance constraint in the regulator synthesis procedure, the regulator is designed based only on the regulation conditions (4.4), (5.2), and (5.3). We have

$$
\begin{aligned}
& C_{Q_{1}}=\left[\begin{array}{lllll}
0 & -1.0448 & 0.4263 & -0.0905 & 0.0975
\end{array}\right] \times 10^{-3}, \\
& C_{Q_{2}}=\left[\begin{array}{lllll}
0 & -0.5360 & -0.5121 & 1.3472 & -1.3250
\end{array}\right] \times 10^{-3} .
\end{aligned}
$$

The experimental results corresponding to the above designed controller are shown in Figures 4, 5, 6, and 7, which include the two cases of with and without the unknown random disturbance $d_{w}$. It can be seen that the resulting system with the designed controller can exactly reject the known disturbance in the presence of switching (contacting), however 




(a)

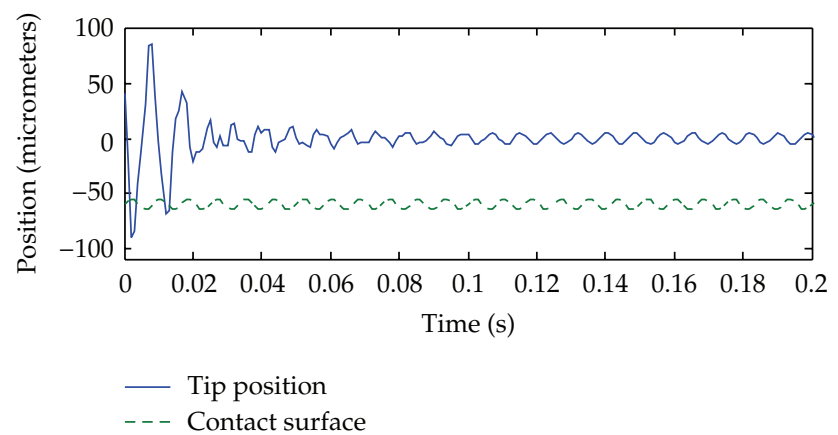

(b)

Figure 4: Experimental results of the performance variable and the positions of the tip and of the contact surface for the case of $d_{w}=0$ and obtained using the controller designed without accounting for the $\mathrm{H}_{2}$ performance constraint.

it cannot attenuate the disturbance $d_{w}$ properly. By considering the $H_{2}$ constraint in the regulator synthesis procedure, we obtain

$$
\begin{gathered}
C_{Q_{1}}=\left[\begin{array}{lllll}
0 & 0.1376 & -0.2452 & 0.1665 & 0.0208
\end{array}\right] \times 10^{-3}, \\
C_{Q_{2}}=\left[\begin{array}{lllll}
0 & 0.1129 & -0.2334 & 0.2105 & -0.1002
\end{array}\right] \times 10^{-3}, \\
r^{2}=0.118 .
\end{gathered}
$$

The experimental results are shown in Figures 8 and 9, which indicate that the disturbance $d_{w}$ has been attenuated effectively and the tip of the suspension beam tracks the contact surface while maintaining the desired separation between the tip and the contact surface.

\section{Conclusion}

The problem of regulation in discrete-time bimodal switched systems against partially known deterministic exogenous inputs and unknown random inputs is treated. A regulator design approach based on the parameterization of a set of stabilizing controllers for the switched system is presented. First, regulation conditions for the switched system are derived based on the Youla parameterization of a set of stabilizing controllers, where a set of $Q$ parameters is constructed to achieve regulation against the deterministic exogenous inputs and also against the unknown random exogenous input. Then, the regulator synthesis algorithm is developed 


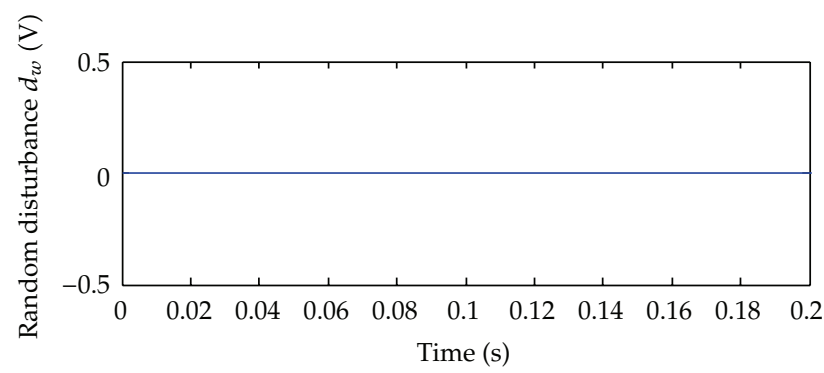

(a)

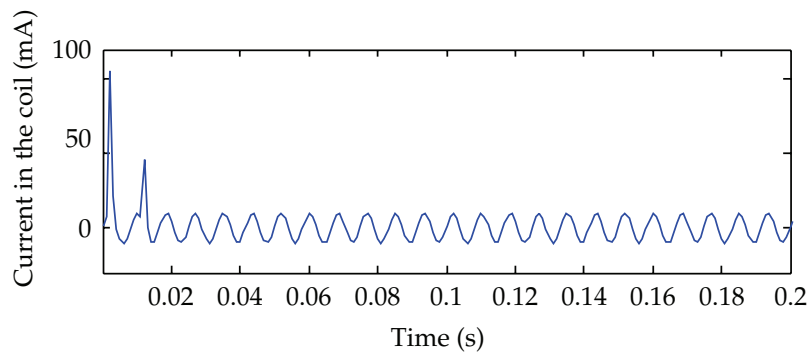

(b)

Figure 5: Experimental results showing the random disturbance $d_{w}$ and the total current in the electromagnetic actuator coil obtained using the controller designed without accounting for the $\mathrm{H}_{2}$ performance constraint.

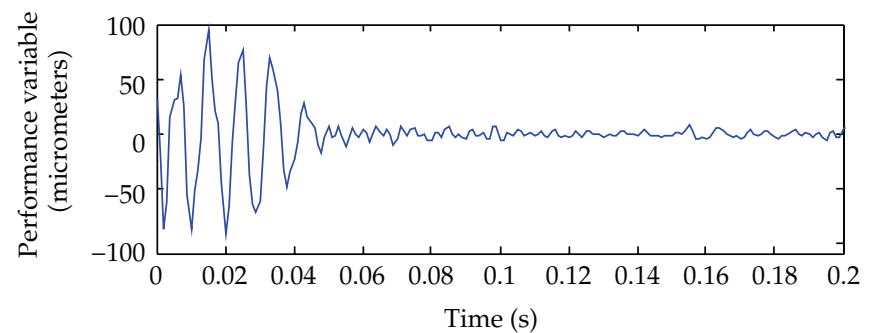

(a)



(b)

Figure 6: Experimental results of the performance variable and the positions of the tip and of the contact surface in the presence of $d_{w}$ and obtained using the controller designed without accounting for the $\mathrm{H}_{2}$ performance constraint. 


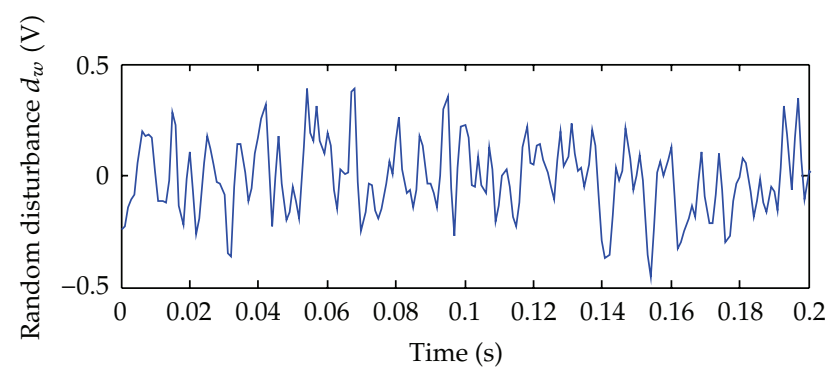

(a)

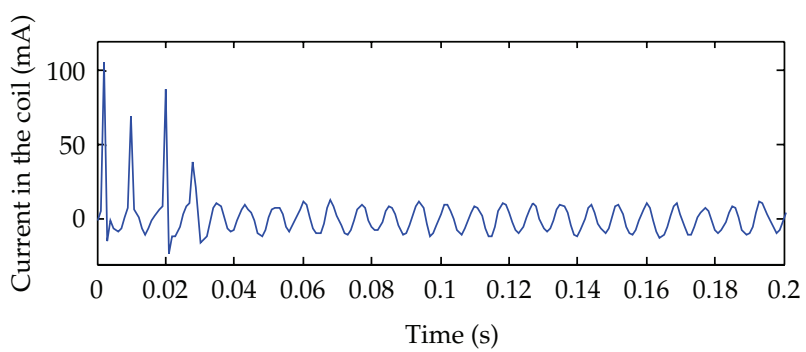

(b)

Figure 7: Experimental results showing the random disturbance $d_{w}$ and the total current in the electromagnetic actuator coil obtained using the controller designed without accounting for the $\mathrm{H}_{2}$ performance constraint.

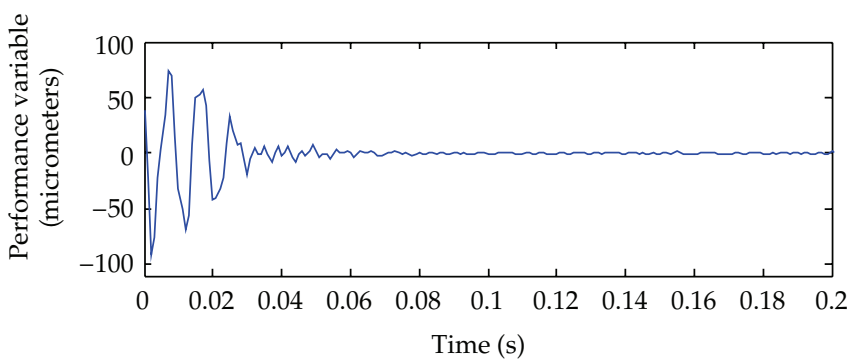

(a)

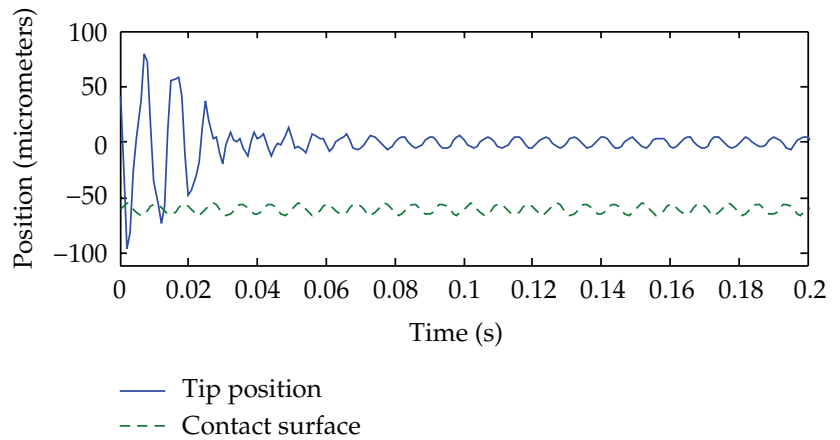

(b)

Figure 8: Experimental results of the performance variable and the positions of the tip and of the contact surface in the presence of $d_{w}$ and obtained using the controller designed by accounting for the $\mathrm{H}_{2}$ performance constraint. 


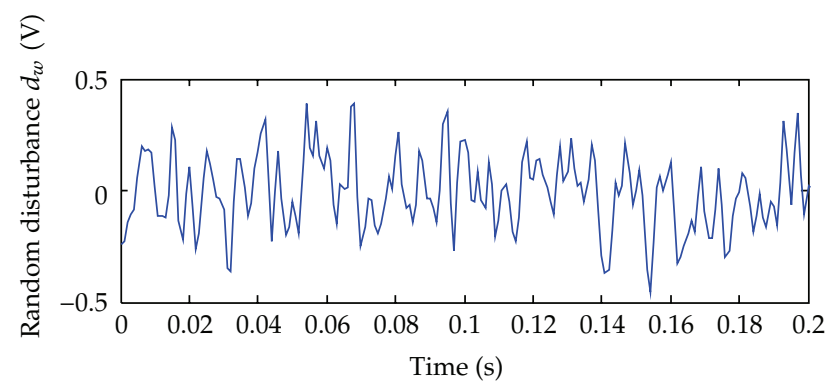

(a)

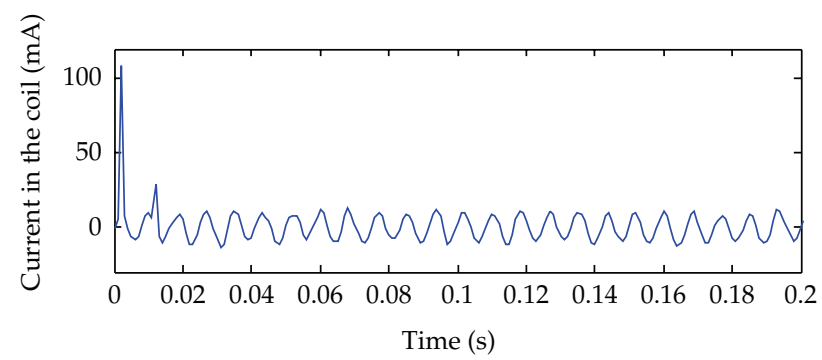

(b)

Figure 9: Experimental results showing the random disturbance $d_{w}$ and the total current in the electromagnetic actuator coil obtained using the controller designed by accounting for the $\mathrm{H}_{2}$ performance constraint.

based on solving a set of properly formulated linear matrix inequalities. Finally, a switched bimodal mechanical system experimental setup involving a flexible beam subject to contact vibrations is used to successfully demonstrate the performance of the proposed regulator.

\section{Acknowledgments}

The authors would like to thank the associate editor and the anonymous reviewers for their comments. This work was supported by the National Natural Science Foundation of China (51075254), the Shanghai Pujiang Program (11PJ1404000), the Innovation Program of Shanghai Municipal Education Commission (11YZ16), and the Natural Sciences and Engineering Research Council of Canada.

\section{References}

[1] D. Liberzon, Switching in Systems and Control, Birkhauser Boston, Boston, Mass, USA, 2003.

[2] Z. Sun and S. S. Ge, Switched Linear Systems-Control and Design, Springer, London, UK, 2005.

[3] E.-K. Boukas, Stochastic Switching Systems: Analysis and Design, Birkhäuser Boston Inc., Boston, Mass, USA, 2006.

[4] J. Wang, M. Feckan, and Y. Zhou, "Ulam's type stability of impulsive ordinary differential equations," Journal of Mathematical Analysis and Applications, vol. 395, no. 1, pp. 258-264, 2012.

[5] K. Linda, K. Ono, and M. Yamane, "Dynamic characteristics and design consideration of a tripad slider in the near-contact regime," Journal of Tribology, vol. 124, pp. 600-606, 2002. 
[6] A. I. Vakis, S. C. Lee, and A. A. Polycarpou, "Dynamic head-disk interface instabilities with friction for light contact (surfing) recording," IEEE Transaction on Magnetics, vol. 45, no. 11, pp. 4966-4971, 2009.

[7] U. Boettcher, H. Li, R. A. de Callafon, and F. E. Talke, “Dynamic flying height adjustment in hard disk drives through feedforward control," IEEE Transactions on Magnetics, vol. 47, no. 7, pp. 1823-1829, 2011.

[8] S. Devasia, B. Paden, and C. Rossi, "Exact-output tracking theory for systems with parameter jumps," International Journal of Control, vol. 67, no. 1, pp. 117-131, 1997.

[9] K. Sakurama and T. Sugie, "Trajectory tracking control of bimodal piecewise affine systems," International Journal of Control, vol. 78, no. 16, pp. 1314-1326, 2005.

[10] N. van de Wouw and A. Pavlov, "Tracking and synchronisation for a class of PWA systems," Automatica, vol. 44, no. 11, pp. 2909-2915, 2008.

[11] Z. Wu and F. Ben Amara, "Parameterized regulator synthesis for bimodal linear systems based on bilinear matrix inequalities," Mathematical Problems in Engineering, vol. 2008, Article ID 341720, 21 pages, 2008.

[12] Z. Wu and F. Ben Amara, "Regulation in bimodal systems," International Journal of Robust and Nonlinear Control, vol. 18, no. 11, pp. 1115-1141, 2008.

[13] Z. Wu and F. Ben Amara, "Regulator synthesis for bimodal linear systems," IEEE Transactions on Automatic Control, vol. 56, no. 2, pp. 390-394, 2011.

[14] B. A. Francis, "The linear multivariable regulator problem," SIAM Journal on Control and Optimization, vol. 15 , no. 3, pp. 486-505, 1977.

[15] K. Zhou, J. Doyle, and K. Glover, Robust and Optimal Control, Prentice Hall, New Jersey, NJ, USA, 1995.

[16] J.-W. Lee and P. P. Khargonekar, “Optimal output regulation for discrete-time switched and Markovian jump linear systems," SIAM Journal on Control and Optimization, vol. 47, no. 1, pp. 40-72, 2008.

[17] J. P. Hespanha, "Root-mean-square gains of switched linear systems," IEEE Transactions on Automatic Control, vol. 48, no. 11, pp. 2040-2045, 2003. 




Advances in

Operations Research

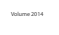

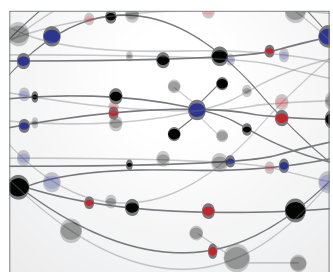

\section{The Scientific} World Journal


International Journal of

Mathematics and

Mathematical

Sciences


Journal of

Applied Mathematics
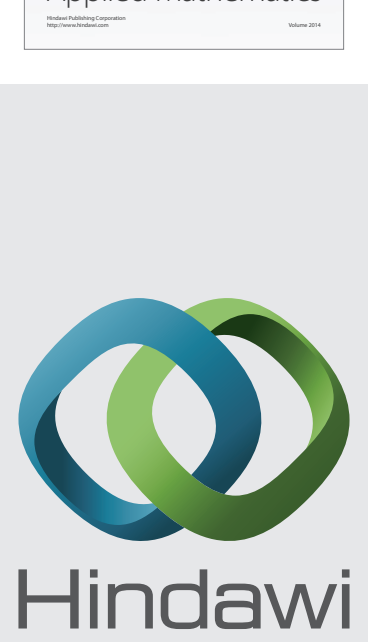

Submit your manuscripts at http://www.hindawi.com
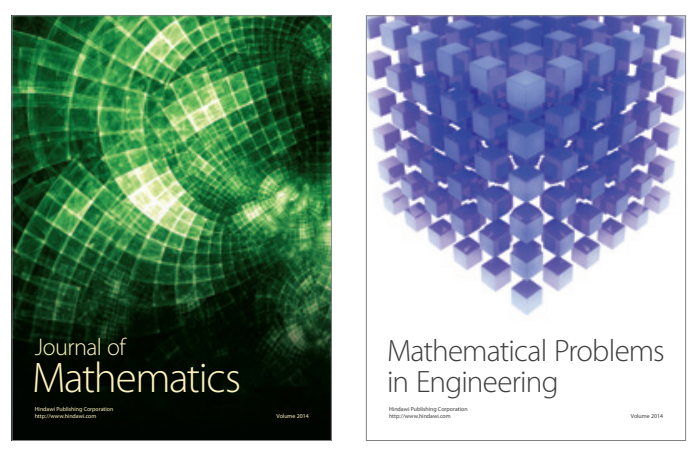

Mathematical Problems in Engineering


Journal of

Function Spaces


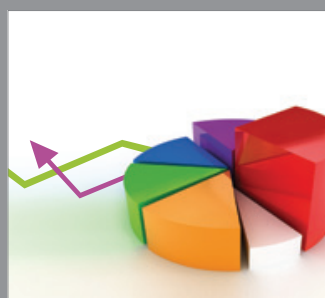

ournal of

Probability and Statistics

Promensencen
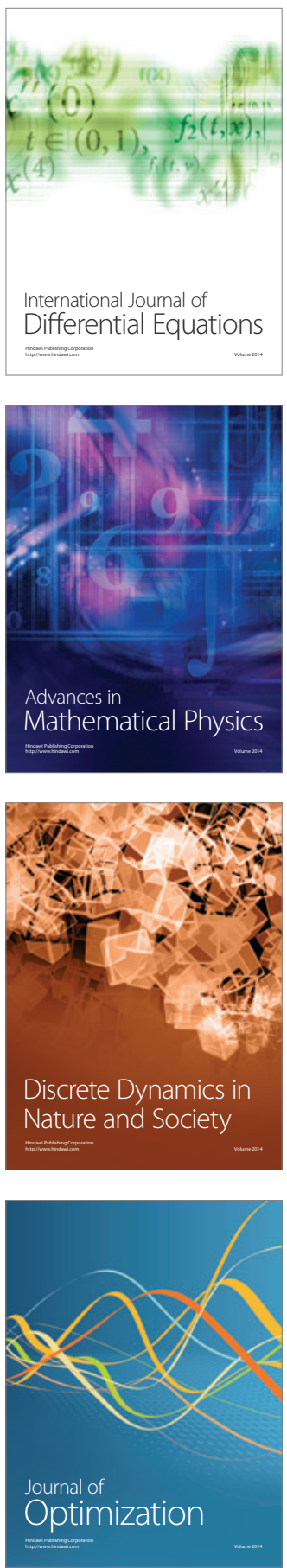